\title{
Relation between Influenza Vaccination and Outpatient Visits, Hospitalization, and Mortality in Elderly Persons with Chronic Lung Disease
}

Kristin L. Nichol, MD, MPH; Leslie Baken, MD; and Andrew Nelson, MPH

Background: Influenza vaccine is underused in groups targeted for vaccination.

Objective: To define the effects of influenza and the benefits of influenza vaccination in elderly persons with chronic lung disease.

Design: Retrospective, multiseason cohort study.

Setting: Large managed care organization.

Patients: All elderly members of a managed care organization who had a previous diagnosis of chronic lung disease.

Measurements: Outcomes in vaccinated and unvaccinated persons for the 1993-1994, 1994-1995, and 19951996 influenza seasons were compared after adjustment for baseline demographic and health characteristics. All data were obtained from administrative databases.

Results: Vaccination rates were greater than $70 \%$ for each season. Among unvaccinated persons, hospitalization rates for pneumonia and influenza were twice as high in the influenza seasons as they were in the interim (noninfluenza) periods. Influenza vaccination was associated with fewer hospitalizations for pneumonia and influenza (adjusted risk ratio, 0.48 [95\% Cl, 0.28 to 0.82$]$ ) and with lower risk for death (adjusted odds ratio, $0.30[\mathrm{Cl}, 0.21$ to 0.43]) during the influenza seasons. It was also associated with fewer outpatient visits for pneumonia and influenza and for all respiratory conditions.

Conclusions: For elderly persons with chronic lung disease, influenza is associated with significant adverse health effects and influenza vaccination is associated with substantial health benefits, including fewer outpatient visits, fewer hospitalizations, and fewer deaths. Health care providers should take advantage of all opportunities to immunize these high-risk patients.

Ann Intern Med. 1999;130:397-403.

From the Veterans Affairs Medical Center and the University of Minnesota Medical School, Minneapolis, Minnesota; and HealthPartners and Group Health Foundation, Bloomington, Minnesota. For current author addresses, see end of text. $\bigoplus$ ach year, influenza and its complications are hospitalizations, tens of thousands of excess deaths, and billions of dollars in health care costs $(1,2)$. Elderly persons and persons with certain underlying medical conditions experience more than $80 \%$ of the serious complications of influenza, such as hospitalization and death $(3,4)$. Among elderly persons, those with chronic lung disease are an especially high-risk group: Their hospitalization rates for pneumonia are 2 to 7 times those of their counterparts without underlying pulmonary conditions (57). Despite long-standing recommendations for annual immunization (4), approximately $35 \%$ of elderly persons are not vaccinated (8). The highest-risk elderly persons, such as those with chronic lung disease, also have inadequate vaccination rates (9).

Persistent uncertainties about the risks of influenza and the benefits of influenza vaccination may contribute to the underuse of influenza vaccine in very high-risk populations, such as elderly persons with chronic lung disease. Some studies, for example, have suggested that vaccine immunogenicity may be impaired in elderly and chronically ill populations $(10,11)$. Little has been published about the clinical impact of influenza or the observed effectiveness of vaccination in persons with specific underlying medical conditions.

Of persons 65 years of age or older, $12 \%$ have chronic lung disease (9). We conducted this cohort study to define the effects of influenza and the benefits of influenza vaccination in this very-highrisk group of chronically ill elderly persons.

\section{Methods}

\section{Setting}

Group Health, a staff-model health maintenance organization, is one of several health care plans affiliated with HealthPartners, which is a vertically integrated health care organization. Group Health has 21 clinics, 350 salaried physicians, and more than 250000 members in the Minneapolis-St. Paul area. In 1989, it adopted a modified version of the Minneapolis Veterans Affairs Flu Shot Program and piloted it in 2 clinics (12). The influenza vaccination program was expanded to include all of the Group 
Health staff clinics in 1990 and has been maintained since then. This successful program has resulted in influenza vaccination rates of $60 \%$ or more for elderly members and has been associated with improved health and decreased medical care costs for these persons (13). Components of the program include standing orders for nurses, walk-in vaccination clinics that complement other clinic-based vaccination efforts, and reminder mailings to high-risk patients.

\section{Cohort}

All Group Health members who 1) were at least 65 years of age on 1 October 1993, 2) had received a diagnosis of chronic lung disease (International Classification of Diseases, Ninth Revision, Clinical Modification [ICD-9-CM], codes 491-496 and 500518) during the previous 12 months, and 3) were alive on the first day of the outcome period were included in the cohort.

\section{Data Collection and Study Outcomes}

This study is one of several projects that will assess the impact of vaccine-preventable diseases and the benefits of vaccination in this cohort of elderly persons with chronic lung disease. We previously reported the results of serial, single-season cohort studies conducted among all elderly members of Group Health $(13,14)$. All of the study data were obtained from the administrative databases of Group Health. Information on the baseline demographic and health characteristics of persons in the cohort was collected for the period from 1 October 1992 through 30 September 1993 and included age, sex, number of physician visits, previous hospitalizations for pneumonia and influenza (ICD-9-CM codes 480-487), and other high-risk diagnoses (diabetes [ICD-9-CM code 250], heart disease [ICD-9CM codes 393-398, 410-414, 425, and 428-429], chronic renal disease [ICD-9-CM codes 581-582 plus Current Procedural Technology, 4th revision (CPT-4), code 39.95 for dialysis], vasculitis or rheumatologic disease [ICD-9-CM codes 446, 710, and 714], dementia or stroke [ICD-9-CM codes 290294, 331, 340-341, 348, and 438], and cancer [ICD9-CM codes 140-208]). Influenza vaccination status (vaccination between 1 October and 31 December for each season [CPT-4 code 907.24]) and pneumococcal vaccination status (vaccination between 1 January 1988 and the first day of each outcome period [CPT-4 code 907.32]) were assessed before each outcome period.

The primary study outcomes were the number of hospitalizations for pneumonia and influenza (ICD9-CM codes 480-487), the number of hospitalizations for all acute and chronic respiratory conditions (ICD-9-CM codes 460, 462, 465, 466, 480-487, 490-
496, and 500-518), and deaths from all causes during the three influenza seasons studied. Secondary outcomes were outpatient visits for pneumonia and influenza and outpatient visits for all acute and chronic respiratory conditions during the influenza seasons. To test the adequacy of the multivariate models, we assessed the rates of two control outcomes: 1) all noncardiopulmonary hospitalizations during the influenza seasons and 2) hospitalizations for pneumonia and influenza during the interim periods. We hypothesized that the rates of both control outcomes would be similar in the two study groups.

\section{Outcome Periods}

Outcomes were assessed from 15 November 1993 through 31 March 1996. The three influenza seasons included in the study were the main periods of interest. The dates for the influenza seasons were selected to span periods of continuous influenza activity in Minnesota and were chosen on the basis of Minnesota Department of Health influenza surveillance information (15-17). The inclusive dates of the influenza seasons were similar for each of the study years. To facilitate data analysis, we therefore selected the same time period-15 November through 31 March - to define each influenza season for 19931994, 1994-1995, and 1995-1996. The two interim periods extended from 1 April through 14 November in 1994 and 1995.

\section{Statistical Analysis}

Baseline demographic and health characteristics in vaccinated and unvaccinated persons were compared by using Student $t$-tests and chi-square tests. We used generalized estimating equations to fit multivariate models so that we could compare risks for the study outcomes over the three influenza seasons or the two interim periods in vaccinated and unvaccinated persons while controlling for covariates and potential confounders (SAS, version 6.12, SAS Institute, Inc., Cary, North Carolina). The numbers of hospitalizations in the two groups were compared by using Poisson regression with repeated measures. To accommodate the assumption of a Poisson distribution, the number of hospitalizations for each person in each period was truncated at 4 (this affected $<0.1 \%$ of persons for pneumonia hospitalizations, $<0.1 \%$ of persons for all noncardiopulmonary hospitalizations, and $<0.5 \%$ of participants for hospitalizations for all respiratory conditions). The risks for death in the two groups during the influenza seasons were compared by using logistic regression with repeated measures. For the numbers of outpatient visits, we used Poisson regression with repeated measures. To accommodate the assumption of a Poisson distribution, we confined the analysis to 
persons who had at least one visit and we truncated the numbers of visits for each person to 8 within each study period (this affected $<0.5 \%$ of persons for outpatient visits for pneumonia and influenza and $<10 \%$ of persons for outpatient visits for all respiratory conditions). To test whether the proportion of persons in the two groups who had at least one outpatient visit differed, we used logistic regression with repeated measures.

In all of these models, vaccination status was included as a time-varying covariate. Age, sex, and influenza and pneumococcal vaccination status were included in all final models. Age was dichotomized ( $<80$ years or $\geq 80$ years), given the skewness of the underlying distribution. All other variables listed in Table 1 were considered for inclusion in the final models because they 1) had previously been reported to be associated with increased risk for at least one of the primary study outcomes, 2) were thought to be clinically relevant, or 3) were variables for which persons studied differed significantly at baseline. Variables were selected after the use of stepwise procedures if they were associated with the outcome with a $P$ value less than 0.2 in the full model, which included all possible variables, and if they also had a $P$ value less than 0.1 in the final, reduced model. We also tested for evidence of interaction between influenza vaccinations and pneumococcal vaccinations. All models were adjusted for the numbers of months of follow-up available for each person in the study. A variable for year was also included in the models. Final models were reviewed to confirm the clinical appropriateness of the variable set. To ensure that the reduced models represented sufficient confounder and covariate groups, we also compared the estimate of vaccination effect seen in the full models with that seen in the reduced models, verifying that no meaningful differences in effect estimates existed between the two models.

For selected outcomes, we estimated the percentage reduction (prevented fraction) associated with vaccination. This was calculated by subtracting the risk ratio from 1 (18). In estimating the percentage reduction in deaths associated with vaccination, we used the odds ratio as an estimate of relative risk because the outcome events were uncommon (19).

\section{Results}

The cohort comprised 1898 persons. Influenza vaccination rates were $72 \%, 74 \%$, and $75 \%$ for the 1993-1994, 1994-1995, and 1995-1996 seasons, respectively. Among persons who had been vaccinated before the third influenza season, $76 \%$ had received immunizations for the two previous seasons and
Table 1. Characteristics of Study Cohort before the First Influenza Season*

\begin{tabular}{|c|c|c|c|}
\hline Characteristic & $\begin{array}{l}\text { Vaccinated } \\
\text { Persons } \\
(n=1366)\end{array}$ & $\begin{array}{l}\text { Unvaccinated } \\
\text { Persons } \\
(n=532)\end{array}$ & $P$ Value \\
\hline Mean age $\pm S D, y$ & $73.5 \pm 5.3$ & $75.0 \pm 6.9$ & $<0.001$ \\
\hline percentiles), $n$ & $72.8(69.4,76.6)$ & $73.5(70.0,79.4)$ & \\
\hline Age $\geq 80$ years, $\%$ & 14.8 & 26.3 & $<0.001$ \\
\hline \multicolumn{4}{|l|}{ Previous diagnoses, \% } \\
\hline Heart disease & 34.4 & 39.5 & 0.04 \\
\hline Diabetes & 16.5 & 18.4 & $>0.2$ \\
\hline Stroke or dementia & 3.4 & 12.0 & $<0.001$ \\
\hline Chronic renal disease & 2.9 & 3.8 & $>0.2$ \\
\hline Rheumatologic disease & 2.8 & 3.4 & $>0.2$ \\
\hline Cancer & 16.5 & 21.4 & 0.01 \\
\hline History of pneumonia, \% & 16.5 & 20.3 & 0.06 \\
\hline Pneumococcal vaccination, \% & 49.6 & 31.2 & $<0.001$ \\
\hline \multirow{2}{*}{$\begin{array}{l}\text { Mean physician visits } \pm S D, n \\
\text { Median physician visits ( } 25 \text { th, } \\
75 \text { th percentiles), } n\end{array}$} & $18.5 \pm 13.1$ & $16.5 \pm 13.3$ & 0.003 \\
\hline & $16.0(9.0,24.0)$ & $13.0(7.2,22.0)$ & \\
\hline
\end{tabular}

* Baseline diagnoses and resource utilization are for the 12-month period from 1 October 1992 through 30 September 1993. Pneumococcal vaccination status was determined for the period from 1 January 1988 through the first day of the outcome period.

$21 \%$ had received one immunization over the two previous seasons. At baseline, persons who were vaccinated before the first influenza season were younger; more likely to be male; more likely to have received a pneumococcal vaccination; and less likely to have a diagnosis of heart disease, cancer, or dementia or stroke. They also had a higher rate of outpatient visits during the previous year (Table 1).

For each of the influenza seasons, significant influenza A/H3N2 activity was seen. There was also significant influenza B activity during the 1994-1995 season and significant influenza A/H1N1 activity during the 1995-1996 season. A good to excellent match was found between vaccine and circulating virus strains for each season (Cox N. Personal communication).

A total of 112 hospitalizations for pneumonia and influenza, 566 hospitalizations for all respiratory conditions, 1113 outpatient visits for pneumonia and influenza, 9517 visits for all respiratory conditions, and 149 deaths occurred during the three influenza seasons studied. A total of 259 persons were disenrolled from the health plan at some time during the outcome period.

The observed annualized incidence rates of hospitalizations for pneumonia and influenza in each study period for vaccinated and unvaccinated persons are shown in the top panel of the Figure; the adjusted risk ratios for hospitalization for unvaccinated persons compared with vaccinated persons by study period are shown in the bottom panel of the Figure. Substantial seasonal differences were seen in the rates of hospitalization for pneumonia for unvaccinated participants, whereas this seasonal variability markedly diminished among vaccinated persons. For unvaccinated persons, the overall annualized 
incidence of hospitalization for pneumonia and influenza was 55 hospitalizations per 1000 personyears (95\% CI, 34 to 76 per 1000 person-years) during the interim periods. This rate doubled to 111 hospitalizations per 1000 person-years (CI, 80 to 141 per 1000 person-years) during the influenza seasons $(P<0.001)$. Among vaccinated persons, on the other hand, the annualized hospitalization rates were 41 per 1000 person-years (CI, 30 to 51 per 1000 person-years) during the interim periods and 45 per 1000 person-years (CI, 33 to 56 per 1000 person-years) during the influenza seasons $(P=0.11)$.

Over the three influenza seasons, influenza vaccination was associated with a $52 \%$ reduction in the numbers of hospitalizations for pneumonia and influenza (adjusted risk ratio [RR], 0.48; $P=0.008$ ) and with a $70 \%$ reduction in risk for death (adjusted odds ratio [OR], 0.30; $P<0.001$ ) (Table 2). The number of hospitalizations for all respiratory conditions did not differ significantly between the two groups (RR, $0.76 ; P=0.13)$. For outpatient visits, influenza vaccination was not associated with a lower risk for having at least one visit for either pneumonia (OR, $0.95 ; P>0.2$ ) or all respiratory conditions (OR, 0.95; $P>0.2$ ) during the influenza seasons (Table 2). However, among persons who had at least one visit, the numbers of outpatient visits were lower for both pneumonia and influenza (RR, 0.64; $P=0.002)$ and for all respiratory conditions (RR, 0.89; $P=0.002)$. The rates of hospital- ization for the two control outcomes were similar in the two study groups (for hospitalizations for pneumonia and influenza during the interim periods: RR, 0.93 [CI, 0.52 to 1.65 ]; $P>0.2$; for hospitalizations for noncardiopulmonary conditions during the influenza seasons: RR, 0.96 [CI, 0.70 to 1.31]; $P>0.2$ ). However, given the wide CIs, we were unable to exclude a clinically relevant difference.

\section{Discussion}

Elderly persons with chronic lung disease have an especially high risk for complications from influenza. In this study, we demonstrate the substantial health benefits associated with influenza vaccination in this population. Over the three consecutive influenza seasons studied, immunization was associated with a $52 \%$ reduction in hospitalizations for all episodes of pneumonia and influenza and a 70\% reduction in deaths from all causes. Vaccination was also associated with fewer outpatient visits for pneumonia and for all respiratory conditions.

Few previous studies have assessed the benefits of influenza vaccination in adults with chronic lung disease. One small cohort study of vaccinated and unvaccinated patients attending an ambulatory clinic (20) showed that among 66 patients with underlying pulmonary disease, vaccination was associated with a $64 \%$ reduction in febrile respiratory illness and a
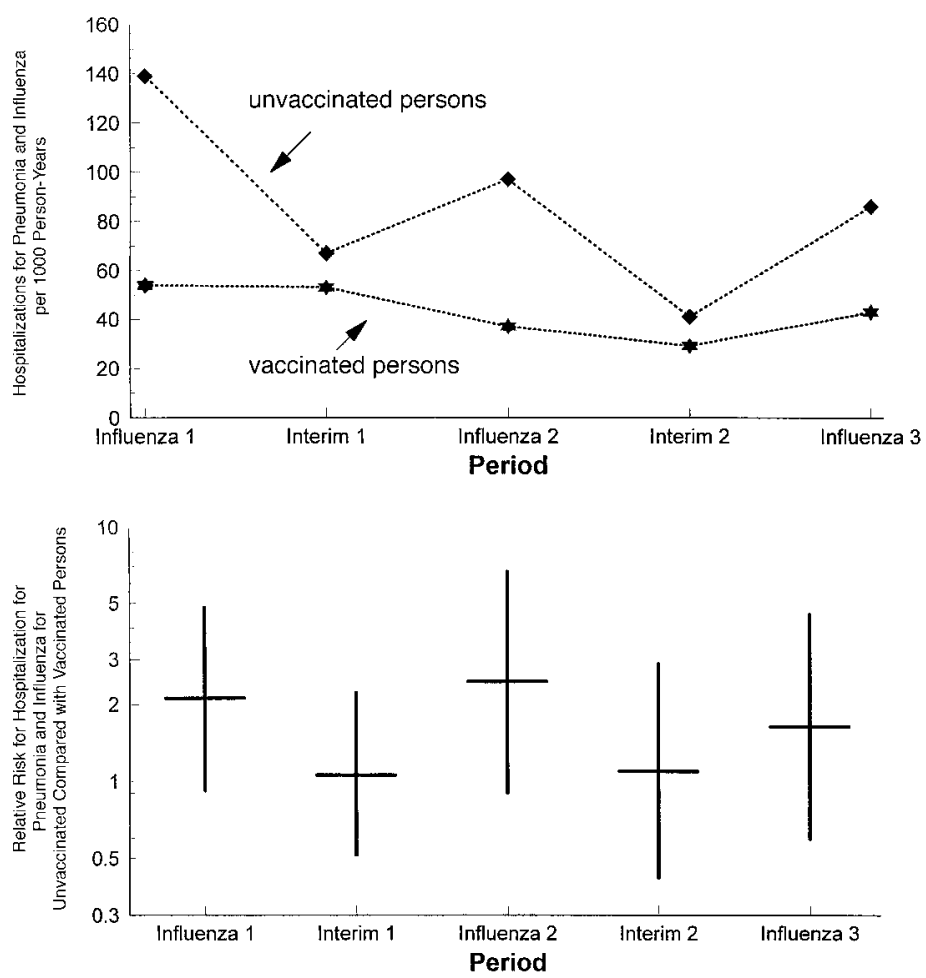

Figure. Hospitalizations for pneumonia and influenza. Top. Observed annualized rates of hospitalization for pneumonia and influenza among vaccinated and unvaccinated persons for each study period. Bottom. Adjusted risk ratios for hospitalization for pneumonia and influenza by study period, comparing unvaccinated with vaccinated persons. The point estimates and $95 \% \mathrm{Cls}$ are shown. 
$77 \%$ reduction in all respiratory illnesses during an influenza A season. Another study of 195 patients who were hospitalized with exacerbations of chronic airflow obstruction during an influenza season (21) showed that $20 \%$ of unvaccinated persons and only $1.4 \%$ of vaccinated persons had evidence of influenza A infection. Our study extends previous observations by showing, in a large cohort and over consecutive seasons, that vaccination of elderly persons with chronic lung disease is associated with significantly fewer outpatient visits, hospitalizations, and deaths due to complications of influenza.

As has been noted elsewhere for elderly persons with chronic pulmonary disease (5-7), our study participants were at especially high risk for hospitalization due to pneumonia and influenza during the influenza seasons: They had hospitalization rates more than four times higher than those of their Group Health counterparts who did not have underlying lung disease (Nichol KL. Personal communication). Nevertheless, the clinical effectiveness of vaccination in our study was similar to that seen in previous studies of unselected elderly persons. Influenza vaccination in a placebo-controlled trial of low-risk older adults reduced rates of clinical and serologic influenza by $58 \%$ (22). Observational studies among noninstitutionalized persons have shown that vaccination of elderly persons is associated with reductions of $30 \%$ to $57 \%$ in hospitalizations for pneumonia and influenza $(6,7,13,23-25)$. Vaccination has also been associated with reductions in deaths from all causes of $27 \%$ to $75 \%(13,24,26)$. Among elderly nursing home residents, vaccination is associated with a $50 \%$ reduction in hospitalizations and a $68 \%$ reduction in deaths during influenza seasons (27).

Among unvaccinated persons, rates of hospitalization due to pneumonia and influenza during the influenza seasons in our study were approximately twice those seen during the interim, noninfluenza periods. Similar excess hospitalization rates have been seen by other investigators during influenza A epidemic years $(5,28,29)$, and smaller increases have been seen during influenza B years (28). This increase over the rates noted during interim (noninfluenza) periods has been used by others as a measure of the impact of influenza $(28,30)$. According to this definition, about half of the hospitalizations due to pneumonia and influenza during the influenza seasons in our study were actually due to influenza. These findings highlight the substantial effect of influenza and its complications on high-risk populations.

The strengths of our study include the use of a narrowly defined cohort of elderly patients in a single managed care health plan (31). We also had large numbers of persons in both study groups, and
Table 2. Risk for Hospitalizations, Death, and Outpatient Visits among Vaccinated and Unvaccinated Persons during Three Influenza Seasons

\begin{tabular}{lcc}
\hline Outcome & $\begin{array}{c}\text { Risk Ratio or } \\
\text { Odds Ratio (95\% } \\
\text { CI })^{*}\end{array}$ & $P$ Value \\
\hline Hospitalizations for pneumonia and influenza & $0.48(0.28-0.82)$ & 0.008 \\
Hospitalizations for all respiratory conditions & $0.76(0.53-1.09)$ & 0.13 \\
$\begin{array}{l}\text { Death } \\
\text { Outpatient visits for pneumonia and } \\
\quad \text { influenza }\end{array}$ & $0.30(0.21-0.43)$ & $<0.001$ \\
$\geq 1$ outpatient visit & $0.95(0.73-1.25)$ & $>0.2$ \\
$\quad$ Number of outpatient visitst & $0.64(0.49-0.84)$ & 0.002 \\
$\begin{array}{l}\text { Outpatient visits for all respiratory conditions } \\
\geq 1 \text { outpatient visit }\end{array}$ & $0.95(0.84-1.07)$ & $>0.2$ \\
$\quad$ Number of outpatient visitst & $0.89(0.83-0.96)$ & 0.002 \\
\hline
\end{tabular}

* Shown are adjusted risk ratios for the results of the Poisson regression analyses and adjusted odds ratios for the results of the logistic regression analyses. Poisson regression with repeated measures was used for outcomes comparing numbers of hospitalizations and outpatient visits, and logistic regression with repeated measures was used izations and outpatient visits, and logistic regression with repeated measures was used outpatient visit. Age, sex, influenza vaccination status, pneumococcal vaccination status, and year were included in each model. Outcomes were also adjusted for the duration of follow-up for each person studied. Other variables included in the final models were as follows. For hospitalizations for pneumonia and influenza: history of pneumonia or influenza. For hospitalizations for all respiratory conditions: history of pneumonia or influenza, diagnosis of heart disease, number of previous physician visits, interaction term for influenza and pneumococcal vaccinations. For death: history of pneumonia or influenza; diagnosis of stroke or dementia, diabetes, or cancer; number of previous physician visits. For at least one outpatient visit for pneumonia and influenza (logistic regression model): history of pneumonia or influenza, diagnosis of renal disease, number of previous physician visits. For number of outpatient visits (among those with $\geq 1$ visit, Poisson regression model): history of pneumonia or influenza, diase wis of heart disease, number of previous physicin diagnosis of heart disease, number of previous physician visits, interaction term for
influenza and pneumococcal vaccinations. For at least one outpatient visit for all respiratory conditions (logistic regression model): history of pneumonia or influenza, diagnosis of renal disease, number of previous physician visits. For number of outpatient visits (among those with $\geq 1$ visit, Poisson regression model): history of pneumonia or influenza, diagnosis of heart disease, number of previous physician visits.

† Risk ratios are for participants who had at least one outpatient visit.

we had high event rates, which provided us with adequate power to detect differences between the groups. To adjust for covariates and potential confounders, we did multivariate analyses adjusting for patients' baseline demographic and health characteristics. Nevertheless, our study has several important limitations that are common to observational studies. At baseline, unvaccinated persons differed from vaccinated persons for important characteristics, and our models may not have adequately adjusted for the important differences between the groups (32). Furthermore, our databases did not include information on other variables, such as lifestyle behaviors (including smoking); functional status; and socioeconomic status, which may be associated with both the decision to receive vaccine and the types of outcomes included in this study. We did, however, test the adequacy of the multivariate models by comparing the hospitalization rates of the study groups for two control outcomes: rates of hospitalization for pneumonia during the interim periods and rates of hospitalization for noncardiopulmonary causes during the influenza seasons. For both of these control outcomes, only slight differences were seen between vaccinated and unvaccinated persons, although the CIs, especially for hospitalizations for influenza and pneumonia, were sufficiently 
wide to prevent us from ruling out clinically important differences during the control periods.

Misclassification of vaccination status may also have occurred, primarily because of failure to capture vaccination status in the administrative databases. If such misclassification were nondifferential, it would have biased the study toward negative findings. However, if it were differential (that is, if it occurred at a different rate among persons with and persons without an outcome event), then the study findings may have been biased in either direction (33). Two extreme cases would occur if vaccination status was misclassified only among unvaccinated persons who did or did not experience an outcome. If it occurred in those who did experience an outcome, our study findings would overestimate the benefits of vaccination, whereas if it occurred in those who did not experience an outcome, our findings would underestimate the benefits of vaccination. Approximately $95 \%$ of the elderly members of this managed care organization, however, reported receiving their influenza vaccinations at one of the organization's clinics (34). Furthermore, the Group Health databases have been shown to be highly accurate in capturing influenza vaccination status, with $93 \%$ agreement between medical records and the computerized databases (Nichol KL. Personal communication). Therefore, we believe that any effect of misclassification would probably have been modest.

Finally, despite the large number of persons included in our study, the CIs around the adjusted risk ratios for differences in hospitalizations for pneumonia and influenza in particular are large. This reflects a smaller number of outcome events or larger variance, or both, than for some of the other outcomes. The point estimates for these outcomes should therefore be interpreted cautiously because they are imprecise.

To conclude, more than 3 million elderly persons in the United States have chronic lung disease and are at especially high risk for complications of influenza. We have shown that vaccination in this very-high-risk group is associated with substantial health benefits. Health care providers should take advantage of every opportunity to immunize these high-risk persons.

Acknowledgments: The authors thank Cynthia Davey, MS, Bruce Lindgren, MS, and Thomas Louis, PhD, of the Division of Biostatistics and the Biostatistics Consulting Laboratory, University of Minnesota School of Public Health, for consultation and help with statistical analysis.

Grant Support: In part by a grant from Group Health Foundation.

Requests for Reprints: Kristin L. Nichol, MD, MPH, Veterans Affairs Medical Center (111), One Veterans Drive, Minneapolis, MN 55417.
Current Author Addresses: Dr. Nichol: Veterans Affairs Medical Center (111), One Veterans Drive, Minneapolis, MN 55417. Dr. Baken: Park Nicollet Clinic, 3800 Park Nicollet Boulevard, St. Louis Park, MN 55416.

Mr. Nelson: HealthPartners Research Foundation, 8100 34th Avenue South, Box 1309, Bloomington, MN 55440.

\section{References}

1. Couch RB. Advances in influenza virus vaccine research. Ann N Y Acad Sci. 1993;685:803-12.

2. Williams WW, Hickson MA, Kane MA, Kendal AP, Spika JS, Hinman AR. Immunization policies and vaccine coverage among adults. The risk for missed opportunities. Ann Intern Med. 1988;108:616-25.

3. Lui KJ, Kendal AP. Impact of influenza epidemics on mortality in the United States from October 1972 to May 1985. Am J Public Health. 1987;77:712-6.

4. Prevention and control of influenza: recommendations of the Advisory Committee on Immunization Practices (ACIP). MMWR Morb Mortal Wkly Rep. 1998;47(RR-6):1-26.

5. Glezen WP, Decker M, Perrotta DM. Survey of underlying conditions of persons hospitalized with acute respiratory disease during influenza epidemics in Houston, 1978-1981. Am Rev Respir Dis. 1987;136:550-5.

6. Foster DA, Talsma A, Furumoto-Dawson A, Ohmit SE, Margulies JR, Arden NH, et al. Influenza vaccine effectiveness in preventing hospitalization for pneumonia in the elderly. Am J Epidemiol. 1992;136:296-307.

7. Ohmit SE, Monto AS. Influenza vaccine effectiveness in preventing hospitalization among the elderly during influenza type A and type B seasons. Int J Epidemiol. 1995;24:1240-8.

8. Influenza and pneumococcal vaccination levels among adults aged $\geq 65$ years—United States, 1997. MMWR Morb Mortal Wkly Rep. 1998;47:797802.

9. Assessing adult vaccination status at age 50 years. MMWR Morb Mortal Wkly Rep. 1995;44:561-3.

10. Beyer WE, Palache AM, Baljet M, Masurel N. Antibody induction by influenza vaccines in the elderly: a review of the literature. Vaccine. 1989;7: 385-94.

11. Gross PA, Quinnan GV Jr, Weksler ME, Setia U, Douglas RG Jr. Relation of chronic disease and immune response to influenza vaccine in the elderly. Vaccine. 1989;7:303-8.

12. Margolis KL, Nichol KL, Wuorenma J, Von Sternberg TL. Exporting a successful influenza vaccination program from a teaching hospital to a community outpatient setting. J Am Geriatr Soc. 1992;40:1021-3.

13. Nichol KL, Margolis KL, Wuorenma J, Von Sternberg T. The efficacy and cost effectiveness of vaccination against influenza among elderly persons living in the community. N Engl J Med. 1994;331:778-84.

14. Nichol KL, Wuorenma J, von Sternberg T. The benefits of influenza vaccination for low-, intermediate-, and high-risk senior citizens. Arch Intern Med. 1998;158:1769-76.

15. Minnesota Department of Health. Annual Summary of Communicable Diseases Reported to the Minnesota Department of Health, 1993. Disease Control Newsletter. 1994;22:29.

16. Minnesota Department of Health. Annual Summary of Communicable Diseases Reported to the Minnesota Department of Health, 1994. Disease Control Newsletter. 1995;23:36.

17. Minnesota Department of Health. Annual Summary of Communicable Diseases Reported to the Minnesota Department of Health, 1995. Disease Control Newsletter. 1996;24:29-30.

18. Rothenberg RB, Hahn RA. Measures of attribution. In: Haddix AC, Teutsch SM, Shaffer PA, Dunet DO, eds. Prevention Effectiveness: a Guide to Decision Analysis and Economic Evaluation. New York: Oxford Univ Pr; 1996:193-201.

19. Zhang J, Yu KF. What's the relative risk? A method of correcting the odds ratio in cohort studies of common outcomes. JAMA. 1998;280:1690-1.

20. Paul WS, Cowan J, Jackson GG. Acute respiratory illness among immunized and nonimmunized patients with high-risk factors during a split season of influenza A and B. J Infect Dis. 1988;157:633-9.

21. Fox R, French N, Davies L, Reid A, Mutton K, Chakraverty P, et al. Influenza immunization status and viral respiratory tract infections in patients with chronic airflow limitation. Respir Med. 1995;89:559-61.

22. Govaert TM, Thijs CT, Masurel N, Sprenger MJ, Dinant GJ, Knottnerus JA. The efficacy of influenza vaccination in elderly individuals. A randomized double-blind placebo-controlled trial. JAMA. 1994;272:1661-5.

23. Mullooly JP, Bennett MD, Hornbrook MC, Barker WH, Williams WW Patriarca PA, et al. Influenza vaccination programs for elderly persons: cost-effectiveness in a health maintenance organization. Ann Intern Med. 1994;121:947-52.

24. Fedson DS, Wajda A, Nicol JP, Hammond GW, Kaiser DL, Roos LL. Clinical effectiveness of influenza vaccination in Manitoba. JAMA. 1993;270: 1956-61.

25. Ahmed AH, Nicholson KG, Nguyen-van Tam JS, Pearson JC. Effectiveness of influenza vaccine in reducing hospital admissions during the 1989-90 epidemic. Epidemiol Infect. 1997;118:27-33.

26. Fleming DM, Watson JM, Nicholas S, Smith GE, Swan AV. Study of the effectiveness of influenza vaccination in the elderly in the epidemic of $1989-90$ using a general practice database. Epidemiol Infect. 1995;115: 581-9.

27. Gross PA, Hermogenes AW, Sacks HS, Lau J, Levandowski RA. The 
efficacy of influenza vaccine in elderly persons. A meta-analysis and review of the literature. Ann Intern Med. 1995;123:518-27.

28. McBean AM, Babish JD, Warren JL. The impact and cost of influenza in the elderly. Arch Intern Med. 1993;153:2105-11.

29. Barker WH. Excess pneumonia and influenza associated hospitalization during influenza epidemics in the United States, 1970-78. Am J Public Health. 1986;76:761-5.

30. Perrotta DM, Decker M, Glezen WP. Acute respiratory disease hospitalizations as a measure of impact of epidemic influenza. Am J Epidemiol. $1985 ; 122: 468-76$
31. Sackett DL, Haynes RB, Tugwell P. Clinical epidemiology: a basic science for clinical medicine. Boston: Little, Brown; 1985:285-321.

32. lezzoni LI. Risk and outcomes. In: lezzoni LI, ed. Risk Adjustment for Measuring Health Care Outcomes. Ann Arbor, Ml: Health Administration $\mathrm{Pr}$ 1994:1-28

33. Kelsey JL, Whittemore AS, Evans AS, Thompson WD, eds. Methods in Observational Epidemiology. 2d ed. New York: Oxford Univ Pr; 1996:341-64.

34. MacDonald R, Baken L, Nelson A, Nichol KL. Validation of self-report of influenza and pneumococcal vaccination status in elderly outpatients. Am J Prev Med. [In press].

Why did the sight of him, talking to Richard, curl her up? He looked what he was, a great doctor. A man absolutely at the head of his profession, very powerful, rather worn. For think what cases came before him-people in the uttermost depths of misery; people on the verge of insanity; husbands and wives. He had to decide questions of appalling difficulty. Yet—what she felt was, one wouldn't like Sir William to see one unhappy. No; not that man.

Virginia Woolf
Mrs. Dalloway

Submitted by:

Frank Davidoff, MD

Submissions from readers are welcomed. If the quotation is published, the sender's name will be acknowledged. Please include a complete citation (along with the page number on which the quotation was found), as done for any reference.-The Editor 